Martorell, B; Vočadlo, L; Brodholt, J; Wood, IG (2013); Strong pre-melting effect in the elastic properties of hcp-Fe under inner-core conditions, Science 342:6157; 466-468.

10.1126/science. 1243651

\title{
ARTICLE
}

\section{Strong pre-melting effect in the elastic properties of hcp-Fe under inner-core conditions}

Benjamí Martorell*, Lidunka Vočadlo, John Brodholt, lan G. Wood,

Department of Earth Sciences, University College London, Gower Street, London, WC1E 6BT, United Kingdom.

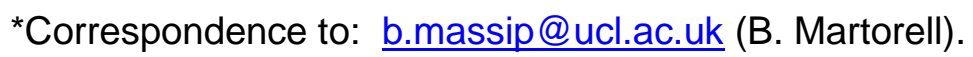

Abstract: The observed shear wave velocity in the Earth's core is much lower than expected from mineralogical models derived from both calculations and experiments. A number of explanations have been proposed, but none sufficiently explain the seismological observations. Using ab initio MD simulations, we obtained the elastic properties of hcp-Fe at $360 \mathrm{GPa}$ up to its melting temperature $\left(T_{m}\right)$. We find that Fe shows a strong non-linear shear weakening just before melting (when $T / T_{m}>0.96$ ), with a corresponding reduction in shear-wave velocity $\left(V_{S}\right)$. Because temperatures range from $T / T_{m}=1$ at the inner-outer core boundary to $T / T_{m} \sim 0.99$ at the center, this strong non-linear effect on $V_{S}$ should occur in the inner core, providing a compelling explanation for the low $V_{S}$ observed.

One Sentence Summary: We find that a strong non-linear shear weakening in hcp-Fe just before melting can explain the low shear velocity observed in the inner core. 
The Earth's inner core is predominantly made of iron but it is commonly assumed to contain 5$10 \% \mathrm{Ni}(1)$ and also light elements such as $\mathrm{Si}, \mathrm{C}$ and S, $2-3 \%$ wt. (1,2). Seismic wave velocities through the inner core are known, but, at present, seismological and mineralogical models for the inner core do not agree (ref. 3-9). A major discrepancy between the observed seismic data and current mineralogical models derived from ab initio calculations is that these mineralogical models predict a shear wave velocity $\left(V_{S}\right)$ that is up to $30 \%$ greater than the seismically observed values $(4,9,10)$. The addition of small quantities of Ni under these conditions does not reduce $V_{S}$ by a sufficient amount to explain this (9), and while the effect of light elements on the velocities of Fe is not totally clear at inner core conditions $(11,12)$, all studies show that light-element effects are too small $\left(<5 \%\right.$ in $\mathrm{V}_{\mathrm{S}}$ for $7 \%$ molar fraction in $\mathrm{Si}$ at $5000 \mathrm{~K}$ and $13000 \mathrm{~kg} \mathrm{~m}^{-3}$, ref. 11) to solve the discrepancy.

Another possible cause of the discrepancy between mineralogical models and seismic data is that the elastic constants of Fe may soften dramatically and non-linearly very near to its melting point $\left(T_{m}\right)$, as has been observed in other metals. For instance, the shear modulus of tin $(\mathrm{Sn})$ has been experimentally and theoretically shown to decrease by more than $50 \%$ at temperatures within about $1 \%$ of its melting point $(13,14)$. According to ab initio simulations, the melting point of pure $\mathrm{Fe}$ at the conditions of the inner core is in the range 6200 to $6900 \mathrm{~K}$ $(15,17)$ using phase coexistence calculations (solid and liquid), with upper limit estimates up to $7500 \mathrm{~K}(18)$ when only the solid phase is heated until melting. The highest temperature for which the elastic properties of hcp-Fe have been obtained computationally is $6000 \mathrm{~K}$ (5); however, relative to the melting point of this simulation, $\mathrm{T} / \mathrm{T}_{\mathrm{m}}$ is probably $\sim 0.8$ and so this temperature is likely to be too low to reveal any strong elastic shear weakening just below melting. 
To examine whether pre-melting effects in the elasticity of Fe can resolve the discrepancy in $V_{S}$ between mineralogy and seismology, we simulated the effect of temperature on $\mathrm{V}_{\mathrm{S}}$ at $360 \mathrm{GPa}$ for hcp-Fe up to its melting point. We performed periodic ab initio calculations based on density functional theory (DFT) derived from quantum mechanics, coupled with molecular dynamics (MD) to obtain the elastic properties of hcp-Fe at finite temperatures (19).

Based on simulations of hcp-Fe at $360 \mathrm{GPa}$ and at temperatures of $6600,7000,7250$ and 7340 $\mathrm{K}(19)$, together with previous simulations at the same pressure and lower temperatures (9), the behaviour of the elastic constants up to $\sim 6600 \mathrm{~K}$ (Fig. 1) is very similar to that found in our earlier work on hcp-Fe at $\sim 315 \mathrm{GPa}$ and temperatures up to $5500 \mathrm{~K}$ (20). Specifically, elastic constants (defined as the ratio of applied stress on a material to the strain produced) $c_{11}, c_{33}$ and $\mathrm{C}_{44}$ decrease with temperature and $\mathrm{c}_{12}$ and $\mathrm{c}_{13}$ slightly increase (Fig. 1). However, one important difference between the simulations at $360 \mathrm{GPa}$ and $315 \mathrm{GPa}$ is that, in the former, $\mathrm{C}_{33}$ is always larger than $c_{11}$, suggesting a large pressure dependence for the $c_{11}-c_{33}$ crossover.

Above $6600 \mathrm{~K}$ our calculations show that all of the elastic constants decrease with temperature, with some of them being very strongly temperature dependent (Fig. 1). In particular, $\mathrm{C}_{44}, \mathrm{C}_{12}$, and $\mathrm{c}_{11}$ drop by 46,19 and $32 \%$, respectively, from 7000 to $7340 \mathrm{~K}$. This pronounced drop for a temperature increase of only $340 \mathrm{~K}$ indicates the calculations above $7000 \mathrm{~K}$ are approaching the melting point of the simulated system. Analysis of the radial distribution functions and the root-mean-square displacements of the atoms, however, confirmed that the system remained completely solid during the simulation at $7340 \mathrm{~K}$ (Fig. S1). A simulation at $8000 \mathrm{~K}$ melted completely after 16 ps (19). 
The temperature dependence of the shear modulus $(G)$ reveals an almost linear decrease up to $7000 \mathrm{~K}$, followed by an abrupt drop beyond this point (Fig. S2). Most models for G vs T describe only the linear region, [for instance the Mechanical Threshold Stress (MTS) model or the Steinberg-Cochran-Guinan (SCG) model $(21,22)]$ and do not describe its behaviour close to the melting temperature. For this reason Nadal and Le-Poac (13) implemented a new model based on Lindemann melting theory which accounts for both the linear region and the region close to the melting temperature. Using this model (19) we obtained a Lindemann coefficient of $f=0.112$ and a melting temperature of $7350 \mathrm{~K}$ for hcp-Fe at $360 \mathrm{GPa}$. The $\mathrm{f}$ coefficient is a materialdependent parameter and is normally between 0.1 and 0.3 (23); hence our value falls in a reasonable range.

We note that the melting temperature obtained in this way is $\sim 850 \mathrm{~K}$ higher than that expected from previous ab initio simulations $(16,17)$ which used the phase coexistence method. This reflects the fact that the goal of the present work is not to obtain an accurate estimate for the melting temperature of hcp-Fe at $360 \mathrm{GPa}$, but rather to investigate the behaviour of its elastic constants (and therefore the seismic velocities) very close to melting. In order to obtain the elastic constants, our simulations needed to be performed on a system with no-pre-existing surface or defects (such as the solid-liquid interface required for the phase coexistence approach), and it is well known that melting temperatures obtained in such a homogeneous system (mechanical melting) are substantially higher than the true thermodynamic (or heterogeneous) melting temperature $(24,25)$. The melting temperature obtained here using the Nadal-Le-Poac model is about $15 \%$ higher than that obtained using free-energies or phase coexistence methods, and is in accord with previous work showing that homogeneous melting temperatures are about 1.2 times the heterogeneous melting temperatures (26-31). 
Although the strong decrease in elastic moduli in Fe observed here is seen close to the homogeneous melting temperature, there is good evidence that this also happens in a real heterogeneous sample. Firstly, the experimentally measured elastic constants of Sn show a strong weakening at a value of $\mathrm{T} / \mathrm{T}_{\mathrm{m}}$ of about 0.99 , where $\mathrm{T}_{\mathrm{m}}$ is the true heterogeneous melting temperature. Secondly, this decrease has also been observed in atmomistic simulations in bcc Vanadium at $\mathrm{T} / \mathrm{T}_{\mathrm{m}}$ of about 0.98 (24). Thirdly, the strong elastic weakening is associated with a rapid increase in defects (defined as over- or under-coordinated atoms) and this occurs at both the homogeneous and heterogeneous melting temperatures (27-31). The only difference in the heterogeneous case is that surface and pre-existing defects can propagate into the bulk at a lower temperature than in a homogeneous solid. The number of atomic defects in our simulation jumps from $34 \%$ at $7340 \mathrm{~K}$ to $70 \%$ at $8000 \mathrm{~K}$ (19, Fig. S3). This is in very good agreement with previous simulations on much larger systems (27-31). Thus it is relative temperature $\left(\mathrm{T} / \mathrm{T}_{\mathrm{m}}\right)$ rather than absolute temperature that is important.

Both compressional $\left(V_{P}\right)$ and shear $\left(V_{S}\right)$ velocities decrease almost linearly with temperature up to $\sim 7000 \mathrm{~K}$ at $360 \mathrm{GPa}$, with a substantial drop beyond this point (Fig. 2). The temperatures at which the velocities from the Preliminary Reference Earth Model (PREM) and our NP-like model agree $\left(7130 \mathrm{~K}\right.$ for $\mathrm{V}_{\mathrm{P}}$ and $7250 \mathrm{~K}$ for $\left.\mathrm{V}_{\mathrm{S}}\right)$ are at $\mathrm{T} / \mathrm{T}_{\mathrm{m}}$ values of 0.971 and 0.988 respectively, relative to the melting temperature of the simulation. Using an adiabatic geotherm (32) we find that the centre of the Earth should be $\sim 200 \mathrm{~K}$ hotter than the inner core boundary (ICB), whereas the melting line at the centre of the inner core is $280 \mathrm{~K}$ above the temperature at the ICB (17); this leads to a value at the centre of the inner core of $T / T_{m}=0.988$. Thus the core does indeed lie in a range of $\mathrm{T} / \mathrm{T}_{\mathrm{m}}$ where the velocities might be expected to be strongly decreased near melting. 
Our results show that $V_{P}$ and $V_{S}$ for the inner core can be fitted with pure Fe for a physically sensible value of $\mathrm{T} / \mathrm{T}_{\mathrm{m}}$. However, our simulated density of pure $\mathrm{Fe}$ is about $3 \%$ too high and so the presence of light elements is still required to match inner-core values (Table S1), but we would expect Fe with a few percent light elements to also show a strong shear softening near the melting temperature. Assuming the light element reduces the melting temperature of the Fealloy, the softening will occur at lower temperatures than in pure $\mathrm{Fe}$, putting it in a more reasonable range of likely core temperatures. However, further investigations into multicomponent systems are essential to fully understand their effect on the elastic properties of the core. Overall, our results demonstrate that the inner core is likely to be in the strongly nonlinear regime and there is no need, therefore, to invoke special circumstances such as strong anelasticity, partial melts or combinations of crystalline phases in order to match the observed seismic velocities and densities of the inner core. 


\section{References and Notes:}

1. F. Birch, Density and composition of mantle and core. J. Geophys. Res. 69, 4377-4388 (1964).

2. J.-P. Poirier, Light elements in the Earth's outer core: A critical review. Phys. Earth Planet. In. 85, 319-337 (1994).

3. A. Cao, B. Romanowicz, N. Takeuchi, An observation of PKJKP: Inferences on inner core shear properties. Science 308, 1453-1455 (2005).

4. L. Vočadlo, Ab initio calculations of the elasticity of iron alloys at inner core conditions: Evidence for a partially molten inner core? Earth Planet. Sc. Lett. 254, 227-232 (2007).

5. X. Sha, R.E. Cohen, First-principles thermal equation of state and thermoelasticity of hcp Fe at high pressures. Phys. Rev. B 81, 094105-10 (2010).

6. X. Sha, R.E. Cohen, Elastic isotropy of $\square$-Fe under Earth's core conditions. Geophys. Res. Lett. 37, L10302-5 (2010).

7. D. Antonangeli et al., Elastic anisotropy in textured hcp-iron to $112 \mathrm{GPa}$ from sound wave propagation measurements. Earth Planet. Sc. Lett. 225, 243-251 (2004).

8. A.P. Kantor et al., Sound wave velocities of fcc Fe-Ni alloy at high pressure and temperature by mean of inelastic X-ray scattering. Phys. Earth Plan. In. 164, 83-89 (2007).

9. B. Martorell, J. Brodholt, I.G. Wood, L. Vočadlo, The effect of nickel on the properties of iron at the conditions of Earth's inner core: Ab initio calculations of seismic wave velocities of Fe-Ni alloys. Earth Planet. Sc. Lett. 365, 143-151 (2013).

10. A.M. Dziewonski, D.L. Anderson, Preliminary reference Earth model. Phys. Earth Planet. In. 25, 297-356 (1981). 
11.D. Antonangeli et al., Composition of the Earth's inner core from high-pressure sound velocity measurements in Fe-Ni-Si alloys. Earth Planet. Sc. Lett. 295, 292-296 (2010).

12.Z. Mao et al., Sound velocities of Fe and Fe-Si alloy in the Earth's core. P. Natl. Acad. Sci. USA 109, 10239-10244 (2012).

13.M.-H. Nadal, P. Le Poac, Continuous model for the shear modulus as a function of pressure and temperature up to the melting point: Analysis and ultrasonic validation. $J$. Appl. Phys. 93, 2472-2480 (2003).

14.M.-H. Nadal, C. Hubert, G. Ravel-Chapuis, Shear modulus determination versus temperature up to the melting point using a laser-ultrasonic device. J. Alloy Compd. 444445, 265-267 (2007).

15.D. Alfè, G.D. Price, M.J. Gillan, Iron under Earth's core conditions: Liquid-state thermodynamics and high-pressure melting curve from ab initio calculations. Phys. Rev. $B$ 65, 165118-11 (2002).

16. D. Alfè, Temperature of the inner-core boundary of the Earth: Melting of iron at high pressure from first-principle coexistence simulations. Phys. Rev. B 79, 060101-4 (2009).

17.E. Sola, D. Alfè, Melting of iron under Earth's core conditions from diffusion Monte Carlo free energy calculations. Phys. Rev. Lett. 103, 078501-4 (2009).

18. G. Morard, J. Bouchet, D. Valencia, S. Mazevet, F. Guyot, The melting curve of iron at extreme pressures: Implications for planetary cores. High Energ. Den. Phys. 7, 141 (2011).

19. RMSD and RDF analysis, Atomic Defect Density, Temperature Evaluation, and Methods are available as Supplementary Materials on Science online. 
20.L. Vočadlo, D. Dobson, I.G. Wood, Ab initio calculations of the elasticity of hcp-Fe as a function of temperature at inner-core pressure. Earth Planet. Sc. Lett. 288, 534-538 (2009).

21. Y.P. Varshni, Temperature dependence of the elastic constants. Phys. Rev. B 2, 39523958 (1970).

22.M.W. Guinan, D.J. Steinberg, Pressure and temperature derivatives of the isotropic polycrystalline shear modulus for 65 elements. J. Phys. Chem. Solids 35, 1501-1512 (1974).

23. D.R. Nelson, (2002) "Defects and geometry in condensed matter physics" (ed. Cambridge Univeristy Press, Cambridge, United Kindom, 2002).

24. V. Sorkin, E. Polturak, J. Adler, Molecular dynamics study of melting of the bcc metal vanadium. I. Mechanical melting. Phys. Rev. B 68, 174102-7 (2003).

25.V. Sorkin, E. Polturak, J. Adler, Molecular dynamics study of melting of the bcc metal vanadium. II. Thermodynamic melting. Phys. Rev. B 68, 174103-9 (2003).

26. K. Lu, Y. Li, Homogeneous Nucleation Catastrophe as a Kinetic Stability Limit for Superheated Crystal. Phys. Rev. Lett. 80, 4474-4477 (1998).

27.F. Delogu, Mecanistic Aspects of Homogeneous and Heterogeneous Melting Processes. J. Phys. Chem. B 110, 12645-12652 (2006).

28. F. Delogu, Cooperative Atomic Displacements and Melting at the Limit of Superheating. J. Phys. Chem. B 110, 3281-3287 (2006).

29.F. Delogu, Homogeneous melting of metals with different crystalline structure. J. Phys: Condens. Matter 18, 5639-5653 (2006). 
30.G. Manai, F. Delogu, Numerical simulations of the melting behaviour of bulk and nanometer-sized Cu systems. Physica B 392, 288-297 (2007).

31.G. Manai, F. Delogu, Homogeneous and heterogeneous melting behaviour of bulk and nanometer-sized Cu systems: a numerical study. J. Mater. Sc. 42, 6672-6683 (2007).

32. J.P. Poirier, "Introduction to the Physics of the Earth's Interior" (ed. Cambridge University Press, Cambridge, United Kingdom, 230-244, 2000).

33. G. Kresse, J. Hafner, Ab initio molecular dynamics for liquid metals. Phys. Rev. B 47, 558-561 (1993).

34.G. Kresse, J. Hafner, Ab initio molecular dynamics for open-shell transition metals. Phys. Rev. B 48,(17):13115-13118 (1993).

35.G. Kresse, J. Hafner, Ab initio molecular-dynamics simulation of the liquid-metalamorphous-semiconductor transition in germanium. Phys. Rev. B 49, 14251-14269 (1994).

36. P.E. Blöchl, Projector augmented-wave method. Phys. Rev. B 50,17953-17979 (1994).

37.G. Kresse, D. Joubert, From ultrasoft pseudopotentials to the projector augmentedwave method. Phys. Rev. B 59, 1758-1775 (1999).

38. J.P. Perdew, Y. Wang, Accurate and simple analytic representation of the electron-gas correlation energy. Phys. Rev. B 45, 13244-13249 (1992).

39.E. Hernández, Metric-tensor flexible-cell algorithm for isothermal isobaric molecular dynamics simulations. J. Chem. Phys. 115, 10282-10290 (2001).

40.I. Souza, J.L. Martins, Metric tensor as the dynamical variable for variable-cell-shape molecular dynamics. Phys. Rev. B 55, 8733-8742 (1997). 
41.G. Simmons, H. Wang "Single crystal elastic constants and calculated aggregate properties: A handbook." (MIT Press, Cambridge, MA, USA, 1971).

42. L. Vočadlo, D. Alfè, M.J. Gillan, G.D. Price, The properties of iron under core conditions from first principles calculations. Phys. Earth Planet. In. 140, 101-125 (2003).

43. H. Flyvbjerg, H.G. Petersen, Error estimates on averages of correlated data. J. Chem. Phys. 91, 461-466 (1989).

Acknowledgments: This work was supported by NERC grant NE/H003975/1 awarded to LV. Calculations were performed in the HECTOR supercomputer facility. Computer code VASP is available at www.vasp.at. The data presented in this paper are given in the main text and in the supplementary materials. BM performed research, analysed data and wrote the paper. LV designed research, analysed data, and wrote the paper. JB analysed data, and wrote the paper. IGW designed the research, analysed data and wrote the paper. 
Fig. 1. Calculated elastic constants for hcp-Fe as a function of simulation temperature at $360 \mathrm{GPa}$. The black solid curves are fits to NP-like models (19). The grey band represents the minimum and maximum melting temperatures (18). The points below $6000 \mathrm{~K}$ are from (9). [A] shows the complete temperature range; $[\mathrm{B}]$ shows results in the non-linear regime.

Fig. 2. Calculated compressional and shear wave velocities for hcp-Fe as a function of the $\mathrm{T} / \mathrm{T}_{\mathrm{m}}$ and simulation temperature at $360 \mathrm{GPa}$. The black solid curves are fits to NP-like models (19). The grey band represents the minimum and maximum melting temperatures for hcp-Fe (18). The points below $6000 \mathrm{~K}$ are from (9). 


\section{Supplementary Materials:}

Methods

Supplementary Text

Equations S1 to S6

Figures S1 to S4

Table S1

References (33-43) 